Revue des patrimoines

Le cheval et ses patrimoines (1ère partie)

\title{
Une campagne nationale : le versement des archives des haras nationaux
}

\section{Pascal Even et Coraline Coutant-Daydé}

\section{(2) OpenEdition}

Journals

Édition électronique

URL : http://journals.openedition.org/insitu/9967

DOI : 10.4000/insitu.9967

ISSN : 1630-7305

Éditeur

Ministère de la culture

Référence électronique

Pascal Even et Coraline Coutant-Daydé, « Une campagne nationale : le versement des archives des haras nationaux », In Situ [En ligne], 18 | 2012, mis en ligne le 20 décembre 2012, consulté le 19 avril 2019. URL : http://journals.openedition.org/insitu/9967 ; DOI : 10.4000/insitu.9967

Ce document a été généré automatiquement le 19 avril 2019

\section{(c) $($ i) $(9)$}

In Situ Revues des patrimoines est mis à disposition selon les termes de la licence Creative Commons Attribution - Pas d'Utilisation Commerciale - Pas de Modification 4.0 International. 


\title{
Une campagne nationale : le versement des archives des haras nationaux
}

\author{
Pascal Even et Coraline Coutant-Daydé
}

1 Nous reconnaissons volontiers, au sein des Archives de France, à la campagne de versement des archives des haras lancée en 2005 un caractère exemplaire. Si l'une des missions fondamentales de l'administration des archives, peut-être la première, consiste à assurer la collecte des matériaux indispensables à l'élaboration de la mémoire du pays, et en premier lieu celle des archives produites par la sphère administrative, il était relativement rare, avant la réforme administrative induite par la révision générale des politiques publiques (RGPP), que des campagnes de versements systématiques soient lancées dans un domaine déterminé, si ce n'est lors des changements de gouvernement avec le versement des archives des ministres et cabinets ministériels. Il a fallu des bouleversements comme celui induit par la révision générale des politiques publiques pour que de telles actions soient lancées. Autre spécificité, les instructions et tableaux de tri que produisent les groupes de travail constitués sous l'égide des Archives de France, pour guider l'action des archivistes et des services producteurs dans la gestion et le tri de leurs archives, se succèdent sans que l'administration des archives ait vraiment la possibilité d'évaluer l'impact exact de ses instructions et qu'elle prenne le temps de vérifier que ses prescriptions ont été suivies d'effet et dans quelles proportions.

De fait, si le versement des archives historiques des haras relancé et encouragé par une instruction conjointe, a entrâné l'entrée dans les services départementaux d'archives d'une quantité importante de documents, cette manne documentaire des haras avait été, d'une manière générale, conservée jusque-là par les établissements, sinon depuis leur création au XVII ${ }^{\mathrm{e}}$ siècle, du moins depuis leur rétablissement par Napoléon. Ce sont donc deux siècles de la mémoire des haras qui sont entrés ainsi dans les services d'archives, tout au moins l'essentiel de cette mémoire car les haras ont connu, comme tous les organismes administratifs, des vicissitudes, des inondations et des incendies ${ }^{1}$. Cette campagne intervenait, rappelons-le, dans le cadre d'une réorganisation profonde des haras nationaux et inaugurait, en quelque sorte, le versement massif des archives des 
établissements publics restructurés puis des services de l'État dans le cadre de la RGPP. Depuis cette date, les services publics d'archives et en premier lieu les archives départementales ont dû accompagner de nombreuses restructurations analogues, celles de la Banque de France, du réseau consulaire des chambres de commerce et d'industrie, des services fiscaux, des hypothèques et du cadastre, des juridictions supprimées pour ne citer que quelques exemples.

\section{Le riche patrimoine écrit des haras}

L'intérêt porté aux archives des Haras par les Archives de France tient également à l'ancienneté de l'institution et à l'importance et à la richesse de leur patrimoine écrit pour la recherche historique. Bien évidemment, ce n'est pas en 2005 que les archivistes ont découvert la richesse des archives des haras et plusieurs versements avaient déjà été enregistrés dans les services d'archives, certains des fonds avaient été même classés et leurs inventaires publiés. Ainsi, dès 1978, le haras de Villeneuve-sur-Lot avait-il versé aux archives départementales du Lot-et-Garonne ses carnets à souche pour la période 1853-1960 ; c'était également le cas des haras de Hennebont, de Gelos, de Saintes et de Pompadour. Il n'en demeure pas moins que l'administration des haras n'avait pas, d'une manière générale, senti la nécessité de déposer les archives de ses établissements dans les services départementaux d'archives, contrairement aux autres entités administratives, et qu'elle avait su, sinon organiser, du moins assurer la gestion de ses archives anciennes. L'une des raisons de ce relatif isolement tenait au demeurant à la place dont disposaient d'une manière générale les haras qui, dans leurs différentes implantations, possédaient suffisamment de vastes locaux, greniers et dépendances, pour conserver leurs archives. Les premières incursions des archivistes dans le domaine des haras étaient par conséquent relativement récentes et ponctuelles même si une fois encore des expériences particulièrement prometteuses avaient été conduites. Le guide des sources de l'histoire du cheval dans les archives publiques françaises publié par les Archives de France en 1993 avait déjà mis en valeur l'importance de ces archives et ouvert bien des pistes de recherche ${ }^{2}$ (fig. $\left.\mathbf{n}^{\circ} \mathbf{1}\right)$. 
Figure 1

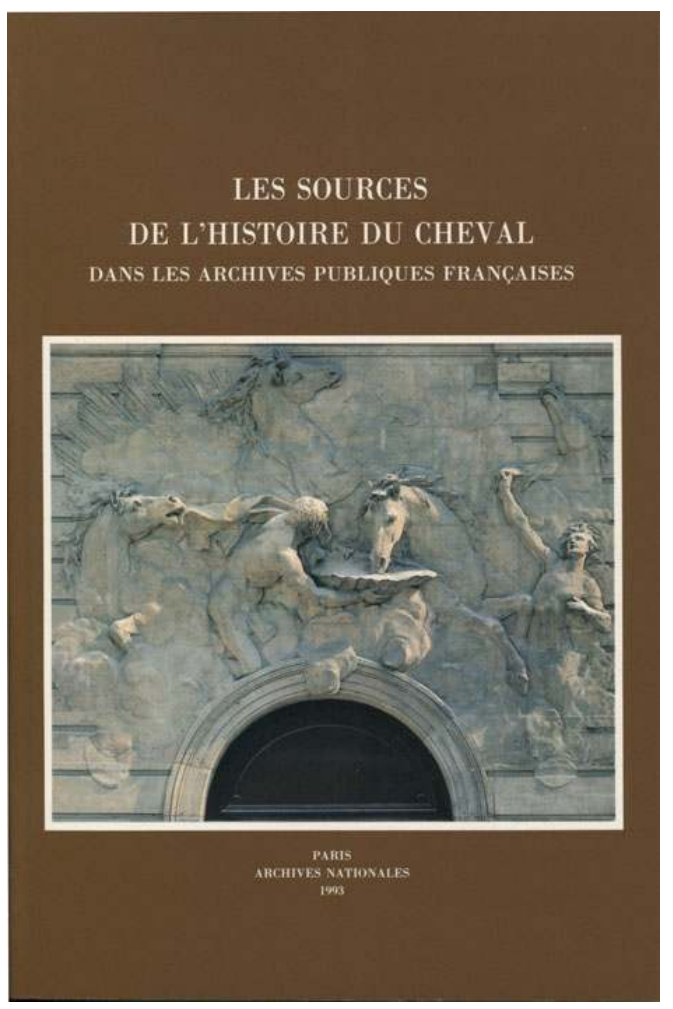

Archives nationales. Les sources de l'histoire du cheval dans les archives publiques françaises. Paris : Archives nationales, 1993.

Mais il est cependant symptomatique que le guide de 1993 ne consacrait que quatorze pages aux archives départementales et encore elles concernaient essentiellement, non les archives des haras eux-mêmes, mais les documents conservés dans les séries des services administratifs en relation avec les haras, intendances et États, administrations de la période révolutionnaire, préfectures et sous-préfectures des $\mathrm{XIX}^{\mathrm{e}}$ et $\mathrm{XX}^{\mathrm{e}}$ siècles. Les auteurs du guide soulignaient la faiblesse des versements « encore très peu nombreux des haras nationaux ou des dépôts d'étalons ». En effet, ils ne citaient que les versements effectués par le dépôt d'étalons d'Hennebont classé par Anne Françoise David et dont l'inventaire venait d'être publié l'année précédente, ainsi que ceux effectués aux archives départementales des Pyrénées-Atlantiques et de Corrèze avec les dossiers des haras d'Arnac Pompadour. Il n'en demeure pas moins que la publication du guide avait permis à la communauté des archivistes de prendre conscience de l'intérêt des archives des haras. À ce titre, cette publication a joué le rôle de révélateur et a facilité à la fois les interventions postérieures des archives dans les haras et ultérieurement la nouvelle campagne de versement engagée à partir de 2005 dont aujourd'hui la communauté scientifique se félicite.

Enfin et surtout, un dernier facteur a joué et explique le succès de cette campagne, c'est le souci également partagé entre les archivistes et les agents des haras de sauvegarder un patrimoine et une mémoire considérés comme en péril. La restructuration et la réforme profonde des haras avaient suscité, comme dans tous les secteurs dont les missions font l'objet d'une réévaluation, un profond désarroi parmi les équipes, désarroi qui ne tenait pas uniquement au bouleversement des traditions de l'institution. Un désarroi tout 
particulièrement perceptible chez les agents conscients de la richesse du patrimoine des haras et soucieux d'éviter sa déperdition. Le traumatisme provoqué par la réforme et la disparition programmée de nombreux établissements avaient fait naître une volonté de sauvegarder un héritage qui risquait, sinon de disparaitre en totalité, du moins d'être sensiblement amoindri. Or les haras conservaient alors et conservent encore un très riche patrimoine non seulement archivistique et documentaire mais encore constitué d'ouvrages imprimés, de documents iconographiques, d'objets mobiliers, de véhicules hippomobiles, d'objets de forge, de sellerie, etc.

\section{Le partenariat entre les Haras et les Archives de France}

6 Ces préoccupations comprises et partagées par les responsables nationaux des haras, se traduisirent par l'adoption d'un programme de conservation patrimoniale et par une demande de conseils formulée auprès du ministère de la Culture. Le partenariat offert par les responsables des haras aux différents services concernés du ministère de la Culture ne pouvait rester sans réponse ; la directrice des Archives de France de l'époque, Martine de Boisdeffre, a souhaité d'emblée donner une réponse positive et accompagner cet effort de sauvegarde comme d'autres services patrimoniaux l'avaient fait avant les archives ou étaient en train de le faire, je songe notamment à l'inventaire des véhicules hippomobiles évoqué au cours du colloque.

7 Et pourtant, lors des premiers contacts entre les archives et les Haras en 2005, à la veille de la commémoration du second bicentenaire de la refondation des haras nationaux par Napoléon, le versement des archives n'apparaissait pas comme une priorité. Les responsables des haras souhaitaient en effet, dans la perspective d'une redéfinition des missions de l'institution, procéder à l'inventaire de leur patrimoine, qu'il s'agisse du patrimoine immobilier et mobilier, des bibliothèques et des archives. Cet inventaire devait précéder une valorisation de ce patrimoine sur site ; à ce titre, des contacts furent pris avec d'une part les conservateurs des antiquités et objets d'art et d'autre part les archivistes afin de faciliter l'élaboration de ces inventaires et la mise en valeur du patrimoine recensé.

8 La note méthodologique élaborée par les haras au cours de l'été et de l'automne 2005 à l'occasion de la mission de recensement engagée en mars de la même année par Philippe de Quatrebarbes et poursuivie par Hugues Jeudy, apparaît, à ce titre, particulièrement instructive : «la finalité de cet inventaire est, dans une première étape, d'identifier les objets mobiliers de toute nature ayant un intérêt artistique, historique ou patrimonial, puis, dans une seconde étape, de réaliser la meilleure valorisation possible de ces objets ». Le versement systématique des papiers anciens des haras dans les services publics d'archives n'était donc pas d'actualité et ce n'est que dans une seconde étape qu'il a paru préférable de confier à des professionnels des archives les fonds des haras dont le traitement, le classement et la communication au public paraissaient difficiles à mettre en œuvre au sein même des établissements qui ne disposaient pas du personnel qualifié indispensable pour le traitement de fonds d'archives aussi anciens et volumineux.

9 C'est en effet lors de la réunion de sensibilisation des responsables des pôles hippiques, organisée le 2 octobre 2005, en présence de la directrice générale des haras, que fut donné le signal de la campagne de versement des archives, l'administration des haras prenant 
soin de rappeler que les haras demeuraient propriétaires des documents versés et que ces derniers pourraient si besoin était, faire l'objet d'une présentation dans les sites. Exemplaires étaient à ce titre les recommandations données alors aux responsables des pôles hippiques dans le domaine des archives: «cette démarche (les demandes de conseils formulées auprès des responsables des archives départementales) permet d'identifier les archives qui présentent un intérêt, d'en assurer la bonne conservation, de les mettre à disposition du public. Les archives départementales publient un inventaire des archives qui leur sont versées, ce qui permet de savoir exactement ce dont on dispose et où le trouver $»$.

Dès le lendemain de la réunion de sensibilisation du mois d'octobre 2005, un groupe de travail était constitué à l'initiative de la direction des Archives de France afin d'élaborer des instructions destinées à permettre l'identification des dossiers des haras présentant un intérêt historique, administratif et juridique et destinés par conséquent à une conservation définitive dans les dépôts d'archives publiques et des papiers dépourvus d'intérêt voués pour leur part à la destruction. Selon la procédure adoptée par les Archives de France et afin d'éviter de prendre, dans le domaine toujours sensible de la sélection, des décisions hâtives mais pourtant irréversibles, le groupe de travail réunissait des archivistes mais également des responsables des haras nationaux, à l'échelon central ainsi qu'à l'échelon territorial, et des historiens du cheval. L'avis de ces derniers apparaissait indispensable pour déterminer le sort final des documents à l'expiration de leur durée d'utilité administrative, en fonction des impératifs de la recherche. Un exercice particulièrement délicat quand on sait combien il est difficile de prévoir les centres d'intérêt futurs de la communauté scientifique, mais un exercice absolument indispensable pour les archivistes qui ne souhaitent pas endosser seuls des décisions définitives en matière d'élimination ou d'échantillonnage. Les travaux de ce groupe aboutirent à l'instruction datée du 16 janvier $2007^{3}$.

\section{L'instruction du 16 janvier 2007}

11 Rappelant le partenariat lancé en novembre 2005 avec les responsables du nouvel établissement public des haras nationaux, l'instruction mettait l'accent sur la sauvegarde des archives des établissements qui n'avaient pas encore procédé au versement de leurs dossiers anciens et dans un souci pédagogique, elle livrait un récapitulatif des versements déjà effectués. L'énumération des établissements ayant déjà procédé au versement, total ou partiel, de leurs archives anciennes, permet au demeurant de mesurer les progrès accomplis en un peu plus de quinze ans dans ce domaine, puisque le guide de 1993 ne mentionnait alors que trois haras ayant versé leurs archives alors qu'ils étaient déjà treize en 2007.

12 L'importance à la fois qualitative et quantitative des fonds des haras déjà collectés soulignait l'intérêt présenté par ces archives et le souci des archivistes d'en assurer la sauvegarde et la mise à la disposition du public. Dans ces conditions, l'instruction de 2007 a certes donné une nouvelle impulsion à la campagne de versement mais elle couronnait une entreprise déjà largement entamée. En revanche, point novateur, elle présentait, par son ampleur nationale, un caractère exhaustif, le but recherché étant de procéder au versement, en quelques années, de l'ensemble des archives historiques des haras.

Il importe d'ajouter que l'élaboration de l'instruction de 2007 est également le résultat de la réflexion engagée par les haras sur la protection et la mise en valeur d'un patrimoine 
mal connu certes mais que personne n'entendait brader. L'on doit dans ces conditions se féliciter tout particulièrement que dans cet effort de sauvegarde patrimoniale, les archives n'aient pas été oubliées mais qu'au contraire elles aient été tout de suite intégrées au programme de protection. Considérées comme une partie importante de l'héritage des haras, au sens donné à ce terme par les anglo-saxons, leur conservation apparaissait aussi évidente et essentielle que celle des bâtiments, des voitures hippomobiles et du mobilier. Mais cette prise en compte ne doit rien au hasard, elle tient en effet à l'effort de sensibilisation engagé depuis des années, aussi bien au niveau des archivistes qu'à celui des professionnels des haras et à la publication du guide des sources. Il nous faut à ce propos adresser des remerciements tout particuliers à ceux et celles qui ont œuvré pour cette véritable prise de conscience. Je voudrais notamment citer Nicole de Blomac, celle qui a sans doute, l'une des premières, révélé aux chercheurs et à la communauté scientifique l'intérêt des archives du cheval et par conséquent celles des haras. Avec son énergie coutumière, Nicole de Blomac avait participé activement à la réalisation du guide des sources de 1993. Depuis lors, chaque fois que l'occasion lui en a été donnée, elle a, de façon militante, œuvré pour sauvegarder et mieux faire connaître les sources documentaires des haras. Qu'elle soit remerciée pour tout le travail accompli.

14 Mais il convient également de ne pas oublier les professionnels des archives qui, au sein du réseau, ont participé à cette campagne et qui se sont mobilisés pour prendre en charge les archives des haras. Bien souvent, les archivistes se félicitent, avec raison, de la cohérence du réseau des archives qui donne plus de visibilité et de force à l'action des archives, mais, dans ce domaine, les encouragements n'ont pas été nécessaires et dès la diffusion de la circulaire, nos équipes d'archivistes ont investi les greniers des haras pour prendre en charge les archives et préparer les versements.

Dans ces conditions, l'instruction sur les archives des haras fait partie, dans le corpus des circulaires des Archives de France, de celles qui ont connu sans doute une application rapide et générale, au point que cinq ans plus tard, il ne demeure plus qu'un établissement, les haras de Rodez, qui n'ait pas versé ses archives ${ }^{4}$. Au total, le bilan peut paraitre impressionnant et personne ne s'en plaindra, surtout pas les chercheurs et les historiens du cheval, même si quelques anomalies subsistent. Assez curieusement, une partie des archives des haras de Strasbourg ont abouti, provisoirement il est vrai, dans les locaux des haras de Tarbes, faute de place aux archives départementales du Bas-Rhin dont le déménagement dans de nouveaux locaux se prépare. Dans tous les autres départements concernés, les dossiers historiques sont maintenant versés et l'organisation même du présent colloque a contribué à l'achèvement de la campagne puisque le directeur des archives départementales des Hautes-Pyrénées a annoncé le récent versement des archives historiques des haras de Tarbes parmi lesquelles au demeurant il a retrouvé des dossiers concernant plusieurs autres départements, ceux des haras de Perpignan entre autres.

16 Enfin, il est rare, faute de temps, que l'administration des archives ait la possibilité de mesurer l'impact réel de ses instructions; pour une fois cependant, nous disposons de données chiffrées précises, le présent colloque nous a donné l'occasion de les établir et nous pouvons ainsi mesurer le succès de la démarche entreprise. La synthèse établie par Coraline Coutant montre suffisamment l'importance du volume collecté et sa richesse.

17 À la suite de l'instruction, les archives départementales concernées ont pris l'attache des services des haras soit pour initier une collecte des archives produites par le haras depuis sa création, soit pour compléter des fonds anciens déjà versés. 

haras par Napoléon, en 1806, mais il faut noter que l'on dispose pour les haras d'ArnacPompadour, d'Hennebont, de Blois et de Rosières-aux-Salines, de documents antérieurs à cette refondation. Les fonds conservés en archives départementales permettent ainsi de parcourir l'histoire de l'institution sur deux siècles, les documents les plus récents remontant au début du XXI ${ }^{\mathrm{e}}$ siècle.

19 Ces fonds sont en général riches et très complets, pour la plupart entre 60 et $90 \mathrm{ml}$. Malheureusement certains fonds ont été partiellement détruits à la suite de sinistre : ainsi le haras de Besançon a brûlé en 1947 et n'a plus guère d'archives anciennes à la suite de cette catastrophe, n'ayant jamais versé de documents aux Archives départementales auparavant. Plusieurs départements au contraire conservent aujourd'hui des séries complètes de carnets de saillies sur plus d'un siècle, tels la Vendée ou la CharenteMaritime. La palme revient aux Archives départementales de la Manche, qui conservent en tout $225 \mathrm{kml}$ pour le haras de Saint-Lô.

20 À ce jour, on peut évaluer les fonds des haras conservés en services publics d'archives à plus de $1200 \mathrm{ml}$.

\section{Des matériaux pour la recherche}

Les chiffres des versements des archives des haras soulignent l'importance des fonds historiques constitués par les haras et la diversité des informations que le chercheur peut y trouver. Il est inutile de revenir sur les descriptions très pertinentes données par le guide des sources de 1993 mais il convient de souligner la variété des sujets de recherche que ces sources permettent, les différentes communications présentées dans le présent colloque l'illustrent suffisamment. Sans prétendre à l'exhaustivité, nous pouvons cependant souligner l'apport considérable de ces archives non seulement pour l'histoire de l'agriculture, pour celle de l'utilisation du cheval à des fins militaires, l'une des vocations premières des haras, mais encore pour celle de l'amélioration de la race chevaline avec notamment les dossiers de gestion des centres de remonte, l'organisation et la réglementation des concours départementaux, régionaux et centraux ou encore la correspondance avec les services vétérinaires.

Dans ce domaine encore, une source remarquable est offerte par les dossiers d'identification des chevaux, les livres généalogiques et la commission du Stud-Book comme aujourd'hui le fichier central des chevaux, le SIRE de Pompadour offrent des sources documentaires de premier plan.

Sur le plan économique et commercial, les dossiers de statistiques de l'élevage équin, les subventions accordées aux syndicats d'élevage, les relations avec les propriétaires, les particuliers, les marchands de chevaux, l'organisation et la production du marché de la viande chevaline, apportent des renseignements tout aussi riches.

Dans le domaine des sports équestres, les chercheurs se régaleront avec les dossiers relatifs à l'équitation de compétition, au tourisme équestre et aux loisirs à cheval. Les dossiers de l'administration centrale comme ceux des haras contiennent des informations complètes sur l'aide consentie aux établissements hippiques, sur l'organisation des prix comme ceux de la Société du Steeple Chase, sur les programmes et le déroulement des épreuves, sur la tutelle des centres de concours, sur les épreuves internationales et olympiques, comme les championnats de France et européens, sur les relations avec la 
Fédération française des sports équestres ou l'École nationale d'équitation. Et que dire des dossiers relatifs à la réglementation des courses, au Pari mutuel, à la répression des fraudes et paris clandestins, à la lutte contre le dopage des chevaux ?

\section{Des fonds mis en valeur par les services d'archives}

Première étape de la mise en valeur des fonds d'archives, l'élaboration d'instruments de recherche et leur publication permettent aux chercheurs de découvrir ces sources inestimables pour l'histoire du cheval. Deux répertoires ont été publiés sous forme traditionnelle papier, ceux qui concernent les archives des haras nationaux d'Hennebont ${ }^{5}$ , en 1992, et de Pompadour ${ }^{6}$, en 2003 (fig. $\mathbf{n}^{\circ}$ 2). Désormais, les instruments de recherche, plus ou moins détaillés, produits par les services d'archives sont publiés sur les sites internet des archives départementales; c'est déjà le cas de la Manche et du Cantal. Les moteurs de recherche permettent également de découvrir sur les sites des archives départementales une riche iconographie sur le cheval, qu'il s'agisse de photographies des chevaux prises par le personnel des haras pour identifier les animaux, de dessins ou de cartes postales.

Figure 2

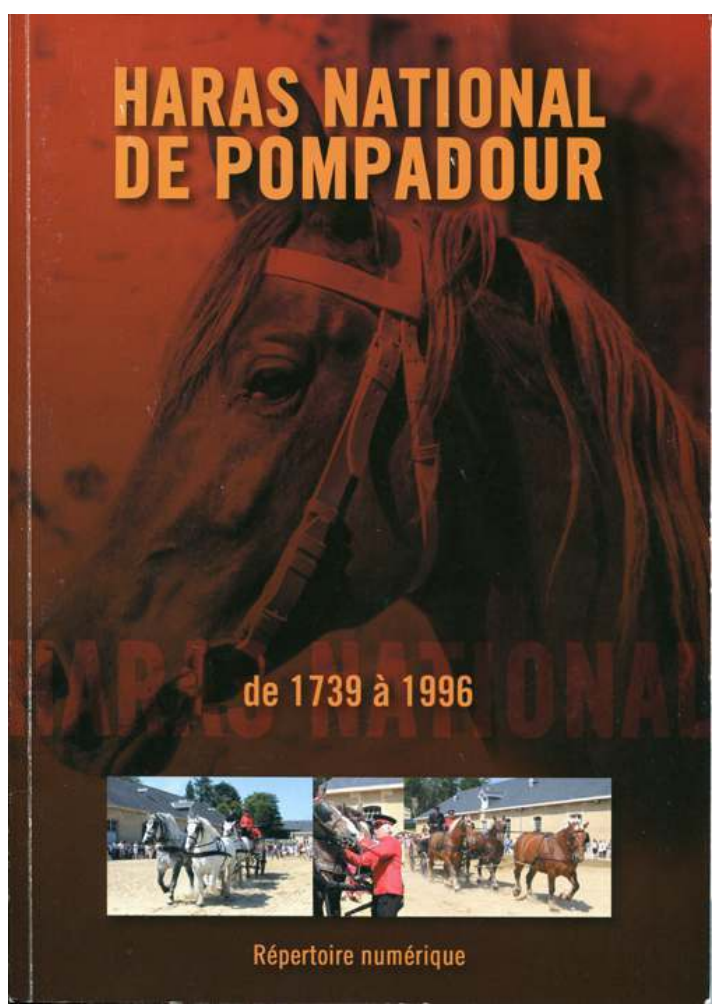

Fonds du Haras national de Pompadour (1739-1996): répertoire numérique de la sous-série 1585 W. Archives départementales de la Corrèze, $2003,104 \mathrm{p}$

Rapidement, et en liaison avec les premiers versements enregistrés en provenance des haras, des services d'archives départementales s'efforcent, dès les années 1980, de faire connaître largement les collections en organisant différentes manifestations destinées au grand public, telles que des expositions sur des thématiques telles que le transport, le commerce ou l'agriculture. Plus près de nous, les archives départementales de la Haute- 
Saône proposent sur leur site internet une exposition virtuelle intitulée « Compagnons de labeur, homme et cheval au travail ${ }^{7}$ » (fig. $\mathbf{n}^{\circ} \mathbf{3}$ ) qui présente les travaux pour lesquels le cheval est indispensable à l'homme (agriculture, mines, halage, etc.). Cette exposition s'accompagne d'un livret pédagogique pour faciliter son exploitation par les enseignants.

Figure 3

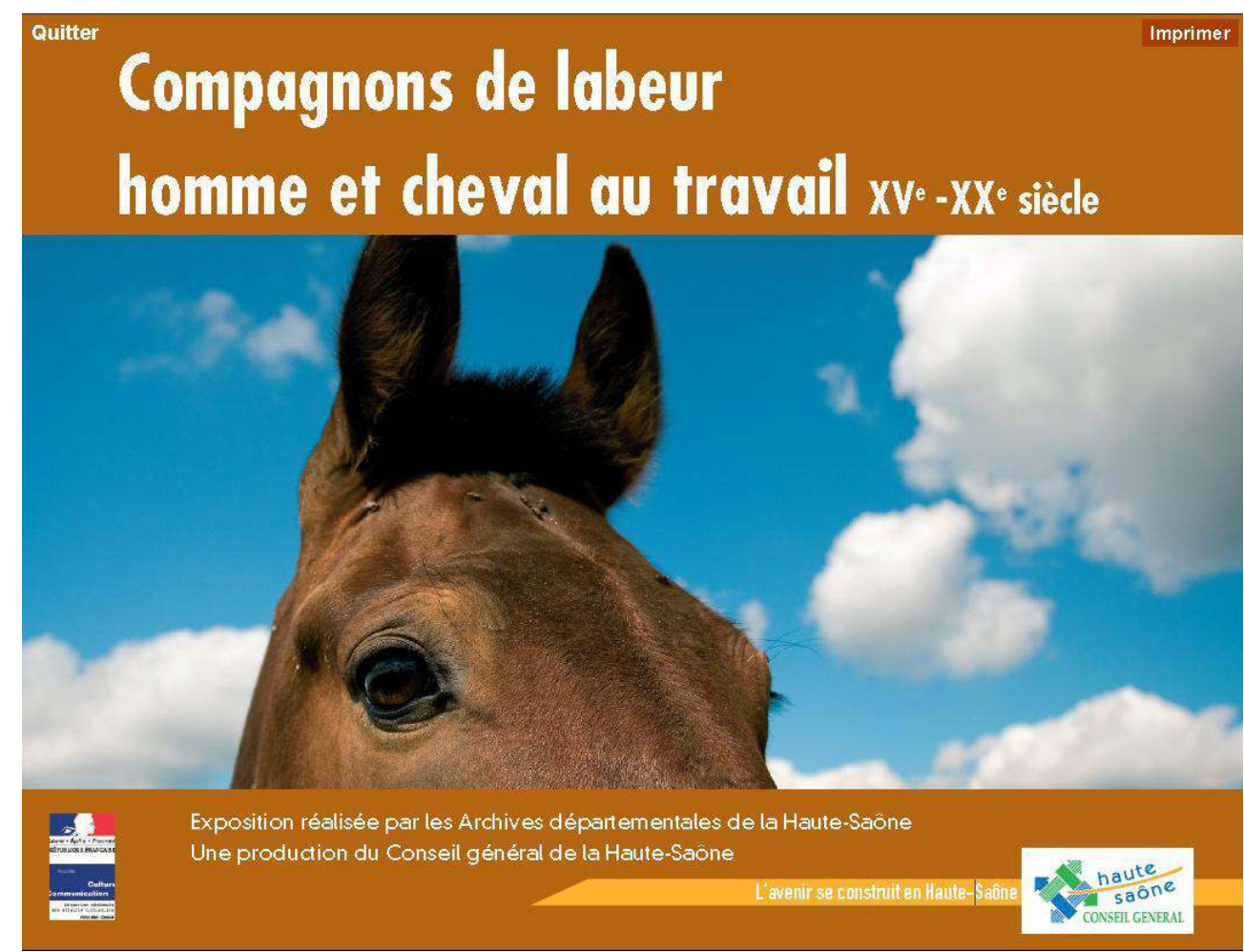

Compagnons de labeur, hommes et cheval au travail, XVe-XXe siècles. Exposition réalisée par les Archives départementales de Haute-Saône, Conseil général de Haute-Saône.

Les archives départementales du Val-de-Marne ont pour leur part réalisé en 2004 "Cheval de Marne ", une exposition présentée à l'Hôtel du département ${ }^{8}$. Notons que le lien entre le Val-de-Marne et les chevaux tient plus à la présence de l'école vétérinaire de Maisons-Alfort et aux courses hippiques qu’à l'élevage équin proprement dit.

Le bicentenaire de la reconstitution des Haras nationaux par Napoléon a constitué en 2006 un événement national, décliné sur l'ensemble du territoire. Les archives départementales de la Manche ont ainsi retracé, dans l'exposition «La Manche, terre du cheval $^{9}$ ", l'histoire des relations étroites entre les Manchois et le milieu du cheval. Une exposition virtuelle a également été créée pour le site internet des archives de la Meurthe-et-Moselle ${ }^{10}$; à travers des documents d'archives conservés dans le service, elle développe quelques considérations zoologiques, raconte la création des haras et illustre les différents usages du cheval et sa place dans la société.

29 Les archives départementales du Morbihan, quant à elles, ont célébré en 2007 le $150^{\circ}$ anniversaire du dépôt d'étalons de la ville d'Hennebont ${ }^{11}$ en proposant sur leur site internet une animation virtuelle retraçant l'histoire du dépôt.

Enfin, un service a particulièrement exploité les fonds du haras et du monde du cheval en général : il s'agit des archives départementales du Doubs, qui ont publié en 2006 Le Cheval comtois $^{12}$ (fig. $\mathbf{n}^{\circ} \mathbf{4}$ ), en collaboration avec l'association Mémoire de l'agriculture comtoise. 
Cet ouvrage très complet retrace l'histoire du cheval en Franche-Comté depuis le XVIII ${ }^{\mathrm{e}}$ siècle, du cheval paysan au cheval guerrier, et des différentes étapes de la reconnaissance de la race de trait. L'ouvrage propose en outre un état des sources sur le cheval comtois, aux archives départementales du Doubs principalement.

Figure 4

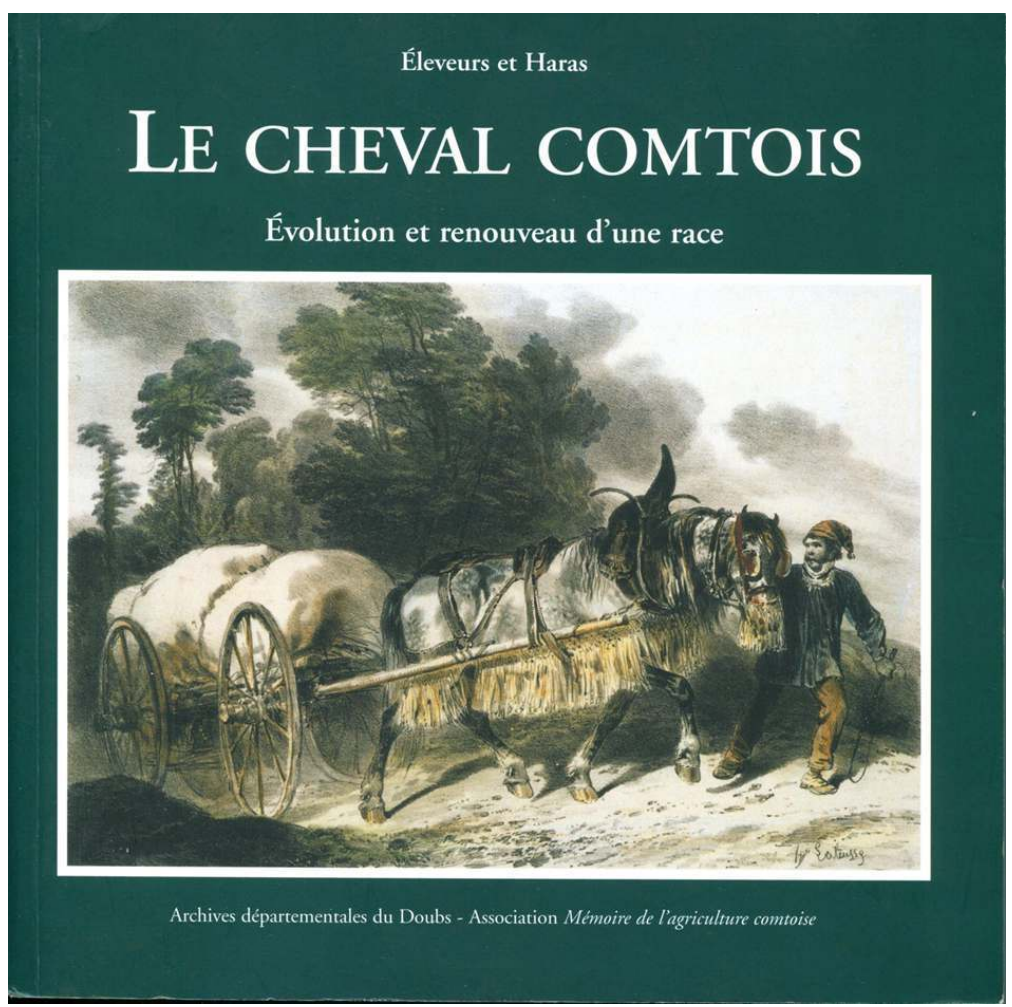

Archives départementales du Doubs et Mémoire de l'agriculture comtoise. Le cheval comtois : évolution et renouveau d'une race : éleveurs et haras. Conseil général du Doubs, 2006.

\section{De nouvelles perspectives}

Il convient de souligner encore la richesse documentaire des dossiers relatifs aux sociétés de courses locales dont beaucoup ont disparu et dont les archives, de nature privée, ont bien souvent été dispersées ou n'ont pas été conservées. Car c'est dans les dossiers des haras que l'on retrouve les dossiers de ces sociétés avec des informations capitales sur leur fonctionnement, leurs responsables, les manifestations organisées par elles et ces dossiers contiennent notamment une riche documentation de nature iconographique. On y retrouve en effet les cartons d'invitation, les programmes et les affiches depuis la fin du $\mathrm{XIX}^{\mathrm{e}}$ siècle, documents susceptibles à eux seuls de participer à la valorisation de ces archives auprès du grand public et d'alimenter des expositions de qualité. C'est la raison pour laquelle les Archives de France souhaiteraient prolonger la campagne de sauvegarde des archives des haras en l'étendant aux papiers des sociétés de courses qui apporteraient un complément indispensable à la vision administrative des concours et épreuves sportives avec des informations sur les relations avec les éleveurs, les propriétaires, les entraîneurs, les jockeys et les lads (fig. $\mathbf{n}^{\circ} 5$ ). 


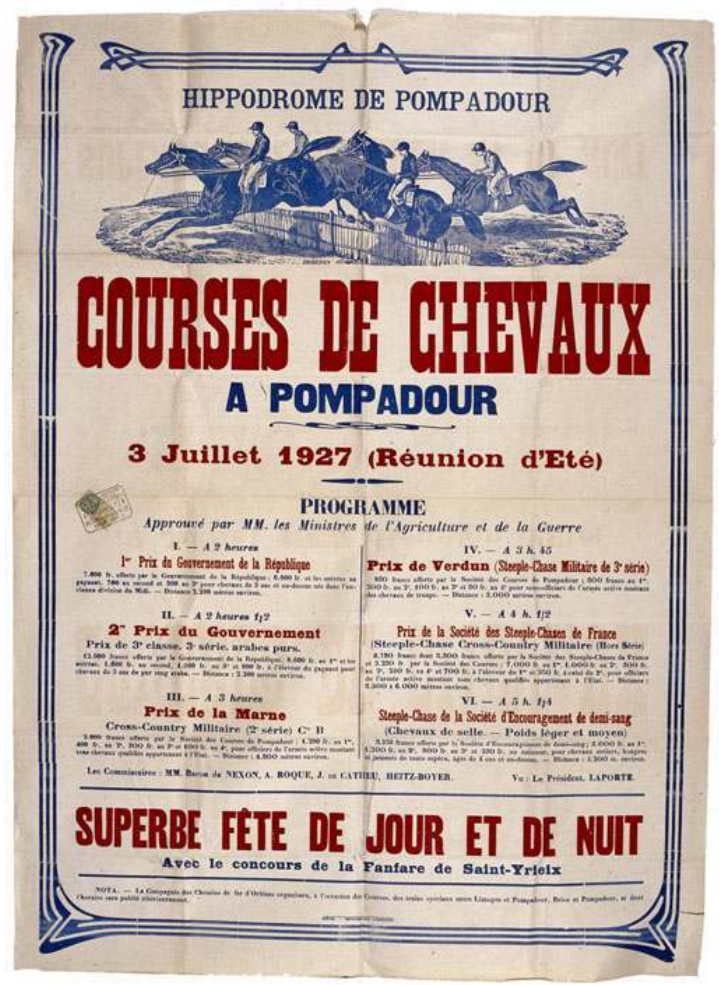

Affiche Courses de chevaux à Pompadour. Archives départementales de Corrèze, FRAD0191Fi1798.

(c) Archives départementales de Corrèze.

Le compte rendu de la réunion du groupe de travail du 2 novembre 2005 soulignait déjà cet objectif et insistait sur la protection des fonds connexes relatifs à l'histoire du cheval, ceux des sociétés hippiques rurales et urbaines, des sociétés de courses, des associations nationales du cheval de race. "Si l'objectif premier du groupe de travail est bien de préparer une instruction de tri et de conservation pour les archives des pôles des haras, il est à espérer que cette mission première contribuera à relancer une politique dynamique de préservation du patrimoine archivistique lié au cheval tant dans le domaine du sport équestre, des courses que de l'élevage ». Dans le même but, l'instruction de 2007 recommandait de prêter une attention particulière aux archives des associations d'éleveurs et des sociétés de concours hippiques. Elle suggérait aux archivistes de solliciter auprès de leurs détenteurs, le dépôt ou le don de ces archives dans les services départementaux d'archives, envisageant, lorsque ces sociétés ont disparu, de les conserver et de les traiter avec les fonds des haras concernés.

On le voit, les pistes de travail sur le cheval et son histoire à partir des archives sont multiples. Les historiens et les chercheurs disposent désormais d'une formidable documentation mise à leur disposition et les différentes communications présentées lors du colloque mettent l'accent sur l'apport de ces sources dans les domaines les plus variés, de l'érudition à la valorisation pédagogique en passant par la conservation de la mémoire orale des haras, domaine dans lequel les archives départementales de la Corrèze disposent d'une expérience précieuse. Le programme de recherche engagé par la direction générale des patrimoines sur trois ans ouvre ainsi des perspectives particulièrement alléchantes et, dans ce programme, nous devons nous féliciter de la part 
prise par le réseau des Archives de France. Qu'il me soit permis, en conclusion, de faire un vœu, celui que le guide des sources de 1993 fasse l'objet d'une actualisation qui fera connaître la richesse de la mémoire écrite des haras.

\section{NOTES}

1. - Les archives des haras de Besançon ont ainsi disparu, pour l'essentiel, lors d'un incendie en 1947.

2. - ARCHIVES NATIONALES. Les sources de l'histoire du cheval dans les archives publiques françaises. Paris : Archives nationales, 1993.

3. - Instruction DPACI/RES/2007/003 du 16 janvier 2007 sur les archives des haras nationaux.

4. - Le versement des archives des haras de Rodez est intervenu au cours de l'année 2012.

5. - DAVID, Anne-Françoise. Répertoire des archives du dépôt d'étalons d'Hennebont. Direction des Archives départementales du Morbihan, 1992, $173 \mathrm{p}$.

6. - Fonds du Haras national de Pompadour (1739-1996) : répertoire numérique de la sous-série 1585 W. Archives

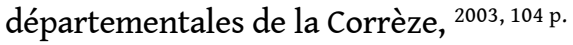

7. - Voir le site : http://archives.cg70.fr/4DCGI/web_Custompage/ cg70_expocompagnonsdelabeur.shtm/ILUMP27466. L'exposition peut également être empruntée aux archives départementales de la Haute-Saône sous forme de panneaux.

8. - Cheval de Marne : exposition, Hôtel du département-Préfecture, Créteil, du 23 juin au 9 juillet 2004. Archives départementales du Val-de-Marne, Créteil, 2005.

9. - Cette exposition peut être visualisée en ligne sur le site internet des archives départementales de la Manche : http://recherche.archives.manche.fr/?id=viewer\&doc=accounts \%2Fmnesys_ad50\%2Fdatas\%2Fir\%2Fimages\%

2FFRAD050_00305.xml\&page_ref=1510\&lot_num=1\&img_num $=1$

10. - Voir le site : www.archives.cg54.fr/Expo/Cheval.htm.

11. - C'est en effet en 1857 que le dépôt d'étalons d'Hennebont a remplacé le haras de Langonnet.

12. - ARCHIVES DÉPARTEMENTALES DU DOUBS ET MÉMOIRE DE L'AGRICULTURE COMTOISE. Le cheval comtois : évolution et renouveau d'une race : éleveurs et haras. Conseil général du Doubs, 2006, $274 \mathrm{p}$.

\section{RÉSUMÉS}

A la fin de l'année 2005, la Direction des Archives de France s'est préoccupée du sort des archives des haras nationaux, qui pour beaucoup n'avaient jamais versé leurs fonds et dont l'administration était alors touchée par la Révision générale des politiques publiques. S'est alors engagée une campagne de collecte généralisée sur l'ensemble du territoire. L'état des lieux des fonds collectés par les services départementaux d'archives montre la mobilisation des services 
départementaux d'archives pour la sauvegarde du patrimoine écrit des haras, dont les actions de valorisation entreprises dans le réseau mettent en lumière la richesse.

At the end of 2005, when the General Public Policy Review engaged the restructuration of the French national stud-farms, the Direction des Archives de France gave attention to their archives, very often managed by their own administration. Then was launched a special campaign for the transfer of this historic archives throughout the country. When we take stock of this operation, we can assess the involvement of the departmental archives services and the exceptional richness of the collections, emphasized by expositions and publications.

\section{INDEX}

Mots-clés : archives, haras, cheval, stud-book, versement, valorisation, expositions, publications

\section{AUTEURS}

\section{PASCAL EVEN}

Chargé de la sous-direction de l'accès aux archives et de la coordination du réseau, service interministériel des Archives de France, ministère de la Culture et de la Communication pascal.even@culture.gouv.fr

\section{CORALINE COUTANT-DAYDÉ}

Sous-direction de l'accès aux archives et de la coordination du réseau, service interministériel des Archives de France, ministère de la Culture et de la Communication coraline.coutant@culture.gouv.fr 